\title{
Avaliação de genótipos de mandioca industriais em área de Cerrado do Noroeste de Minas Gerais ${ }^{1}$
}

\author{
Eduardo Alano Vieira ${ }^{2}$, Josefino de Freitas Fialho ${ }^{3}$, Luiz Joaquim Castelo Branco Carvalho ${ }^{4}$, \\ Juaci Vitoria Malaquias ${ }^{5}$, Francisco Duarte Fernandes ${ }^{6}$
}

http://dx.doi.org/10.1590/0034-737X201562050005

\section{RESUMO}

A avaliação agronômica de cultivares é passo inicial para a determinação do potencial de cultivo de uma cultura em determinado local. Entretanto, ainda não foram desenvolvidos estudos sistemáticos visando à determinação do potencial produtivo de diferentes genótipos de mandioca para indústria, na região de Unaí, Minas Gerais. Por isso, o objetivo deste estudo foi avaliar, por duas safras, 12 genótipos-elite de mandioca para indústria de farinha e fécula, em área de Cerrado da Região Noroeste de Minas Gerais, no município de Unaí. Os experimentos foram conduzidos entre novembro de 2010 e maio de 2012 (safra 2010/2012) e entre novembro de 2011 e maio de 2013 (safra 2011/2013), em área experimental da Escola Agrícola de Unaí. Aos 12 meses após o plantio, nas duas safras, efetuou-se a poda da parte aérea, a $10 \mathrm{~cm}$ do solo, de todos os genótipos. Aos 18 meses após o plantio dos experimentos, os genótipos foram avaliados quanto aos caracteres peso da parte aérea, produtividade de raízes, percentagem de amido nas raízes e foi estimado o rendimento de amido. Os dados foram submetidos à análise de variância individual e conjunta e ao teste de agrupamento de médias. Os resultados revelaram que os genótipos diferiram quanto aos caracteres avaliados, sendo que todos apresentaram características com potencial para cultivo, nas condições locais de avaliação, como elevada produtividades de raízes, elevado peso da parte aérea, elevados teores de amido nas raízes e elevado rendimento de amido, com destaque para o acesso BGMC 996.

Palavras-chave: Manihot esculenta Crantz, melhoramento genético, competição de cultivares, fécula, farinha.

\section{ABSTRACT}

\section{Assessment of cassava genotypes for industry in the Cerrado of Northwestern Minas Gerais}

The assessment of agronomic performance of cultivars is the first step to determine the potential for growing a crop in a particular area. However, systematic studies have not been conducted in order to determine the production potential of different genotypes of cassava for industry in the region of Unaí, Minas Gerais. Therefore, the aim of this study was to evaluate twelve elite genotypes of cassava for industry in relation to agronomic performance and starch content variation during two harvest seasons in the Northwest region of Minas Gerais, in the municipality of Unai. Field experiments were performed in November 2010 and May 2012 (season 2010/2012) and November 2011 and May 2013 (season 2011/2013) in field plots located at the Agricultural School of Unai. Tweive months after planting, in each season, plant shoots were pruned to $10 \mathrm{~cm}$ from the soil. Eighteen months after planting the

\footnotetext{
Submetido em 30/04/2014 e aprovado em 16/07/2015

${ }^{1}$ Trabalho desenvolvido com apoio financeiro da Embrapa, Fundação Banco do Brasil e CNPq

${ }^{2}$ Embrapa Cerrados, Planaltina, Distrito Federal, Brasil. eduardo.alano@embrapa.br

${ }^{3}$ Embrapa Cerrados, Planaltina, Distrito Federal, Brasil. josefino.fialho@embrapa.br

${ }^{4}$ Embrapa Cenargen, Brasília, Distrito Federal, Brasil. luiz.carvalho@embrapa.br

${ }^{5}$ Embrapa Cerrados, Planaltina, Distrito Federal, Brasil. juaci.malaquias@embrapa.br

${ }^{6}$ Embrapa Cerrados, Planaltina, Distrito Federal, Brasil. francisco.duarte@embrapa.br

*Autor para correspondência: eduardo.alano@embrapa.br
} 
experimental plots, the genotypes were evaluated by collecting data on shoot weight, storage root yield, percentage of starch and estimation of starch yield. Data were subjected to analysis of individual and joint variance and mean test group. The results showed that the genotype variation was highly significant for all traits, all genotypes showed characteristics with potential for crops in the local conditions, as indicated by the high yield of storage roots, high weight of shoots, high starch content and high yield of starch with emphasis on genotype BGMC 996.

Key words: Manihot esculenta Crantz, plant breeding, cultivar competition, starch, cassava flour.

\section{INTRODUÇÃO}

A grande maioria das raízes de mandioca (Maninhot esculenta Crantz) produzidas no Brasil destina-se a dois segmentos industriais distintos, as farinheiras e as fecularias. O primeiro é bastante pulverizado e nele predominam unidades industriais familiares, de pequeno porte, enquanto o segundo, o das fecularias, está concentrado nos estados do Paraná, Mato Grosso do Sul, São Paulo e Santa Catarina e, normalmente, apresenta grande capacidade de produção. Entretanto, nesses estados, a cultura da mandioca vem sofrendo elevada competição de outras culturas por áreas de plantio, como a soja, o milho e a canade-açúcar (Vilpoux, 2008), o que aponta no sentido da necessidade da diversificação das áreas de cultivo de mandioca para fins industriais, no Brasil, quer seja para a produção de farinha ou de fécula.

A região do Cerrado que ocupa cerca de $23 \%$ do território brasileiro é apontada como uma das últimas fronteiras agrícolas mundiais e apresenta características de clima e de solo favoráveis ao cultivo de mandioca (Fialho et al., 2013). Por isso, esse bioma é uma alternativa para a diversificação das áreas de produção de mandioca no Brasil. Apesar de todo o potencial da região do Cerrado brasileiro para o cultivo de mandioca, atualmente apenas 10\% da área plantada e 10\% da produção nacional da mandioca estão localizadas na Região, com uma produtividade média de apenas $13 \mathrm{tha}^{-1}$, em uma área colhida de 242.528 ha (IBGE, 2011).

Dentre os fatores responsáveis pela baixa produtividade da mandioca na região, destacam-se o baixo profissionalismo dos produtores, que normalmente não seguem as recomendações para o cultivo, por desconhecimento ou por dificuldades financeiras, o que ocasiona baixa remuneração para a atividade, além da ausência de trabalhos sistemáticos de transferência de tecnologia de produção, de pesquisas em sistemas de produção e de seleção de variedades adaptadas às condições locais.

No município de Unaí, localizado na região Noroeste de Minas Gerais, a mandioca pode ser uma alternativa para a diversificação de cultivos, uma vez que o município apresenta forte produção agropecuária, em seus 3.593 estabelecimentos rurais (IBGE, 2011), destacando-se as produções de milho e de soja, em empreendimentos de grande porte, e a produção de leite e de produtos de subsistência, em empreendimentos de pequeno e de médio portes (Xavier et al., 2005; Oliveira et al., 2009).

Entretanto, ainda não foram desenvolvidos estudos sistemáticos, visando à determinação do potencial produtivo de diferentes genótipos de mandioca para indústria, nessa região. Estudos esses que são o passo inicial para a determinação do potencial real de cultivo da cultura em determinado local e para a possível indicação de algum genótipo para o plantio comercial. Estudos desenvolvidos na região do Cerrado do Brasil Central vêm apontando no sentido do elevado potencial produtivo da cultura na região, tanto no que se refere a genótipos de mandioca para mesa (Fialho et al., 2009; Vieira et al., 2009; Vieira et al., 2011) quanto para indústria (Otsubo et al., 2009; Sagrilo et al., 2010; Vieira et al., 2013). Entretanto, foi detectado um baixo potencial produtivo para acessos de mandioca açucarados (Vieira et al., 2008).

Por essa razão, o objetivo deste estudo foi avaliar agronomicamente, por duas safras, 12 genótipos-elite de mandioca para indústrias de farinha e de fécula, em área de Cerrado da Região Noroeste de Minas Gerais, no município de Unaí.

\section{MATERIAL E MÉTODOS}

Os experimentos de campo foram realizados entre novembro de 2010 e maio de 2012 (safra 2010/2012) e entre novembro de 2011 e maio de 2013 (safra 2011/ 2013), em área experimental da Escola Agrícola de Unaí, localizada no município de Unaí em Minas Gerais.

No período de condução dos experimentos, nas safras 2010/2012 e 2011/2013, respectivamente, as médias das temperaturas máximas diárias foram de 31,55 ${ }^{\circ} \mathrm{C}$ e $31,87^{\circ} \mathrm{C}$, as médias das temperaturas mínimas diárias foram de 18,87 e $19,57^{\circ} \mathrm{C}$, as médias de umidade relativa do ar foram de 63,88 e $63,79 \%$, as velocidades médias dos ventos foram de $1,56 \mathrm{~m} \mathrm{seg}^{-1}$ e $1,60 \mathrm{~m} \mathrm{seg}^{-1}$ e as precipitações acumuladas foram de $2223 \mathrm{~mm}$ e 2167 $\mathrm{mm}$. Nos locais de condução dos experimentos, nas sa- 
fras 2010/2012 e 2011/2013, a composição física e química dos solos foi de, respectivamente, percentagem de argila 70 e $71 \%$, percentagem de silte 25 e $24 \%$, percentagem de areia 5 e $5 \%, \mathrm{pH} \mathrm{em} \mathrm{H}_{2} \mathrm{O} 5,10$ e $5,20, \mathrm{Al}^{+++}$ $0,80 \mathrm{cmol}_{\mathrm{c}} \mathrm{dm}^{-3}$ e $0,40 \mathrm{cmol}_{\mathrm{c}} \mathrm{dm}^{-3}, \mathrm{Ca}^{++} 1,20 \mathrm{cmolc} \mathrm{dm}^{-3}$ e $2,20 \mathrm{cmol}_{\mathrm{c}} \mathrm{dm}^{-3}, \mathrm{Mg}^{++} 1,00 \mathrm{cmol}_{\mathrm{c}} \mathrm{dm}^{-3}$ e $1,20 \mathrm{cmol}_{\mathrm{c}}$

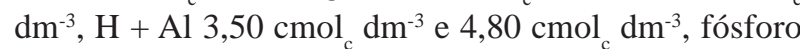
$1,10 \mathrm{mg} \mathrm{dm}^{-3}$ e $1,60 \mathrm{mg} \mathrm{dm}^{-3}$, potássio $261 \mathrm{mg} \mathrm{dm}^{-3} \mathrm{e}$ $203 \mathrm{mg} \mathrm{dm}^{-3}$ e percentagem de matéria orgânica $2,50 \mathrm{e}$ $3,90 \%$.

Os experimentos foram delineados em blocos casualizados, com três repetições. Foram avaliados 12 genótipos-elite de mandioca para indústria, sendo uma variedade e quatro clones do programa de melhoramento de mandioca da Embrapa Mandioca e Fruticultura Tropical, uma variedade e cinco clones do programa de melhoramento genético de mandioca do Instituto Agronômico de Campinas (IAC) e uma variedade local da região do Cerrado, mantida no Banco Regional de Germoplasma de Mandioca do Cerrado (BGMC) (Tabela 1 ).

As parcelas experimentais foram compostas por quatro linhas com dez plantas, em espaçamento de 1,20 m entre linhas e $0,80 \mathrm{~m}$ entre plantas, sendo a área útil de cada parcela constituída pelas 16 plantas centrais. Os tratos culturais (adubação e calagem) foram realizados de acordo com as recomendações técnicas da cultura da mandioca para a região do Cerrado (Fialho et al., 2013, Fialho \& Vieira, 2013) e o controle do mato foi realizado por meio de capina. Aos 12 meses após o plantio, nas duas safras, efetuou-se a poda da parte aérea a $10 \mathrm{~cm}$ do solo, de todos os genótipos.

Aos 18 meses após o plantio dos experimentos, foram avaliados peso da parte aérea, sem a cepa, em $\mathrm{Kg}$ ha${ }^{1}$ (PPA); produtividade de raízes, em $\mathrm{Kg} \mathrm{ha}^{-1}(\mathrm{PR})$, per- centagem de amido nas raízes, por meio do método da balança hidrostática (AM), descrito por Grosmann \& Freitas (1950) e foi estimado o rendimento de amido em $\mathrm{Kg} \mathrm{ha}^{-1}$ (RA), com base na produtividade de raízes e na percentagem de amido nas raízes.

Os dados obtidos foram primeiramente submetidos à análise de variância individual, para determinação da homogeneidade das variâncias dos erros experimentais entre as safras. Atendido o pressuposto de homogeneidade de variâncias dos erros experimentais, relação entre o maior e o menor quadrado médios do resíduo das análises de variância individuais dos caracteres inferiores a sete (Gomes, 1991), foi procedida a análise de variância conjunta dos experimentos. As médias das variáveis respostas foram agrupadas por meio do teste Scott \& Knott, a 5\% de probabilidade de erro. As análises estatísticas foram realizadas com os programas estatísticos SAS (Statistical Analysis System - versão 9.1.3) (SAS Institute 2006) e Genes (Cruz, 2001).

\section{RESULTADOS E DISCUSSÃO}

Uma vez que os quocientes obtidos por meio da relação entre o maior e o menor quadrado médios do resíduo das análises de variância individuais dos caracteres revelaram valores inferiores a sete, indicando a existência de homogeneidade nas variâncias dos erros experimentais, procedeu-se à análise conjunta dos dados (Gomes, 1991).

A análise de variância conjunta mostrou a existência de diferenças significativas entre as médias das safras 2010/2012 e 2011/2013 para o caráter peso da parte aérea (PPA) (Tabela 2), indicando que a média desse caráter foi influenciada pelo fator safra. Entretanto, o fator safra não influenciou significativamente a média dos caracteres produtividade de raízes (PR), teor de amido

Tabela 1: Genótipos de mandioca para indústria analisados com respectivos códigos do banco regional de germoplasma do Cerrado (BGMC) e, ou, de programa de melhoramento, nomes comuns e locais de coleta ou procedência (LCP)

\begin{tabular}{lll}
\hline Genótipos & \multicolumn{1}{c}{ Nome comum } & \multicolumn{1}{c}{ LCP } \\
\hline BGMC 991 & Clone 03 & CNPMF - Cruz das Almas (BA)* \\
BGMC 993 & BRS Formosa & CNPMF - Cruz das Almas (BA) \\
BGMC 994 & Clone 06 & CNPMF - Cruz das Almas (BA)* \\
BGMC 996 & Clone 09 & CNPMF - Cruz das Almas (BA) \\
BGMC 1304 & Clone 9661/06 & CNPMF - Cruz das Almas (BA) \\
BGMC 436 & IAC 12-829 & IAC - Campinas (SP)** \\
Clone 2/87 & Sem denominação comum & IAC - Campinas (SP) \\
Clone 2/88 & Sem denominação comum & IAC - Campinas (SP) $)^{* *}$ \\
Clone 3/88 & Sem denominação comum & IAC - Campinas (SP) $)^{* *}$ \\
Clone 6/88 & Sem denominação comum & IAC - Campinas (SP) \\
Clone 10/87 & Sem denominação comum & IAC - Campinas (SP) \\
BGMC 1371 & Sem denominação comum & Chapada Gaúcha-MG \\
\hline
\end{tabular}

*= Programa de melhoramento genético de mandioca da Embrapa Mandioca e Fruticultura Tropical, baseado em Cruz das Almas $($ BA $) ;{ }^{* *}=$ Programa de melhoramento genético de mandioca do Instituto Agronômico de Campinas (IAC), baseado em Campinas (SP). 
nas raízes (AM) e rendimento de amido (RA) (Tabelas 2 e 3). Apesar de o fator safra, neste trabalho, não ter influenciado os caracteres PR, AM e RA, é importante que em trabalhos futuros seja testado o fator safra para genótipos de mandioca para indústria, uma vez que, em experimento conduzido no Noroeste do Paraná, por três safras, Vidigal Filho et al. (2000) relataram a influência desse fator na manifestação das médias dos caracteres AM, PPA e PR. Por sua vez, Vieira et al. (2009), ao avaliarem genótipos de mandioca para mesa, em seis áreas de Cerrado do Distrito Federal, por duas safras, detectaram a existência de significância para o fator safra para o caráter PR em apenas um dos locais em que os experimentos foram conduzidos.
Dentre os caracteres estudados foi detectada a presença de interação significativa entre os fatores safra e genótipos, para todos os caracteres, com exceção do caráter teor de amido nas raízes (AM) (Tabelas 2 e 3). Esses resultados concordam parcialmente com os de Vidigal Filho et al. (2000) que relatam a existência de interação significativa entre os fatores safras e genótipos também para o caráter AM, o que se pode dever ao fato de, no experimento em que foi detectada interação, os genótipos terem sido avaliados por três safras.

Esses resultados indicam que a ordem de classificação dos acessos para os caracteres PPA, PR e RA foi influenciada pelo efeito safra, em função de os acessos

Tabela 2: Resumo da análise de variância conjunta, média e coeficiente de variação (CV\%) dos caracteres dos caracteres peso da parte aérea, sem a cepa, em Kg ha ${ }^{-1}$ (PPA), produtividade de raízes, em $\mathrm{Kg} \mathrm{ha}^{-1}$ (PR), percentagem de amido nas raízes (AM) e rendimento de amido, em Kg ha ${ }^{-1}$ (RA), avaliados em 12 genótipos de mandioca para indústria, nas safras 2010/2012 (S1) e 2011/2013 (S2), em Unaí-MG

\begin{tabular}{lccccc}
\hline \multirow{2}{*}{ FV } & GL & \multicolumn{3}{c}{ QM } \\
\cline { 3 - 5 } & & PPA & PR & AM & RA \\
\hline Blocos/Safra (B/S) & 4 & 35834714 & 7742483 & 2,13 & 1261343 \\
Safras (S) & 1 & $366586477^{*}$ & 974408 & 0,002 & 128694 \\
Genótipos (G) & 11 & $415326290^{*}$ & $233144975^{*}$ & $9,62^{*}$ & $26291805^{*}$ \\
G x S & 11 & $58498317^{*}$ & $57546749^{*}$ & 2,61 & $5330199^{*}$ \\
Resíduo (R) & 44 & 7361224 & 6814049 & 1,67 & 888555 \\
Total & 71 & & & 30,73 & 10194 \\
Média & & 27274 & 33125 & 4,21 & 9,25 \\
CV (\%) & & 7,95 & & \\
\hline
\end{tabular}

*significativo a $5 \%$ de probabilidade de erro pelo teste $\mathrm{F}$.

Tabela 3: Significância (\%) pelo teste F para o desdobramento da interação acesso x safra dos caracteres dos caracteres peso da parte aérea, sem a cepa, em Kg ha ${ }^{-1}$ (PPA), produtividade de raízes, em Kg ha ${ }^{-1}$ (PR), percentagem de amido nas raízes (AM) e rendimento de amido, em Kg ha ${ }^{-1}$ (RA), avaliados em 12 genótipos de mandioca para indústria, nas safras 2010/2012 e 2011/2013, em Unaí-MG

\begin{tabular}{|c|c|c|c|c|c|}
\hline Fator & GL & PPA & $\mathbf{P R}$ & $\mathbf{A M}$ & $\mathbf{R A}$ \\
\hline Safras & 1 & 3 & 90 & 98 & 88 \\
\hline Genótipos & 11 & 0 & 1 & 2 & 1 \\
\hline Genótipos x safras & 11 & 0 & 0 & 14 & 0 \\
\hline Genótipos dentro da safra 2010/2012 & 11 & 0 & 0 & 0 & 0 \\
\hline Genótipos dentro da safra 2011/2013 & 11 & 0 & 0 & 0 & 0 \\
\hline Safras dentro do clone 10.87 & 1 & 25 & 9 & 14 & 47 \\
\hline Safras dentro do BGMC 436 & 1 & 94 & 33 & 62 & 53 \\
\hline Safras dentro do BGMC 1371 & 1 & 8 & 69 & 53 & 98 \\
\hline Safras dentro do clone 2.87 & 1 & 89 & 92 & 65 & 90 \\
\hline Safras dentro do clone 2.88 & 1 & 68 & 80 & 33 & 80 \\
\hline Safras dentro do clone 3.88 & 1 & 19 & 4 & 56 & 6 \\
\hline Safras dentro do clone 6.88 & 1 & 3 & 24 & 43 & 48 \\
\hline Safras dentro do BGMC 1304 & 1 & 0 & 53 & 80 & 53 \\
\hline Safras dentro do BGMC 991 & 1 & 0 & 18 & 0 & 59 \\
\hline Safras dentro do BGMC 993 & 1 & 2 & 6 & 27 & 3 \\
\hline Safras dentro do BGMC 994 & 1 & 3 & 56 & 56 & 84 \\
\hline Safras dentro do BGMC 996 & 1 & 0 & 0 & 44 & 0 \\
\hline Resíduo & 44 & & & & \\
\hline Total & 71 & & & & \\
\hline
\end{tabular}

Rev. Ceres, Viçosa, v. 62, n.5, p. 453-459, set-out, 2015 
terem apresentado respostas diferenciadas às mudanças ambientais, justificando a necessidade da avaliação dos acessos por mais de uma safra, para a obtenção de uma estimativa mais segura da expressão desses caracteres, como já havia sido relatado, para mandioca, por Borges et al., 2002; Vidigal Filho et al., 2000; Vieira et al., 2009, e a necessidade de se considerar a interação genótipo x safra quando da seleção de determinado genótipo.

Dentre os genótipos avaliados, apenas o genótipo BGMC 991 apresentou média de peso da parte aérea (PPA) superior à dos demais, na safra 2010/2012. Já na safra 2011/2013 o BGMC 991, BGMC 996 e o clone do IAC 3/88 tiveram os melhores PPA em relação aos dos demais (Tabela 4). Os genótipos BGMC 996 e BGMC 991 já se haviam destacado quanto ao PPA, em experimento conduzido por Vieira et al. (2013), em Planaltina-DF. Esse caráter é importante no momento da seleção de genótipos por estar relacionado com a possibilidade de utilização da parte aérea de mandioca como fonte de proteína na alimentação animal (Souza et al., 2011) e cobertura do solo (controle de erosão, manutenção de umidade do solo e controle de ervas daninhas) (Fukuda et al., 2002; Ceballos et al., 2004), em especial para a região de UnaíMG que apresenta forte expressão na produção de leite (Xavier et al., 2005; Oliveira et al., 2009), podendo-se constituir em mais uma opção de geração de renda a partir do cultivo da mandioca.

Quanto à percentagem de amido nas raízes (AM), na safra 2010/2012 os acessos que apresentaram médias significativamente superiores às dos demais foram
BGMC 996, clone 6/88, BGMC 993, clone 2/88, BGMC 994, BGMC 436, clone 2/87 e BGMC 1304 e na safra 2011/2013 foram os acessos BGMC 996 e BGMC 993 (Tabela 4), sendo que os únicos acessos que se destacaram em ambas as safras foram BGMC 996 e BGMC 993 e que os genótipos BGMC 1304 e BGMC 993 já se haviam destacado quanto ao AM, em experimento conduzido por Vieira et al. (2013), em Planaltina-DF. O teor de amido das raízes é um dos caracteres mais importantes para a seleção de genótipos de mandioca para indústria em função de, juntamente, com a produtividade de raízes, determinar os potenciais produtivos de farinha e de fécula de determinado genótipo (Fukuda et al., 2002; Ceballos et al., 2004).

Na safra 2010/2012, o acesso BGMC 996 apresentou médias estatisticamente superiores às dos demais para o caráter produtividade de raízes $(\mathrm{PR})$. Já na safra 2011/2013 os acessos BGMC 994, BGMC 1371, BGMC 996, BGMC 991 e BGMC 993 apresentaram melhores resultados (Tabela 4). O genótipo BGMC 996 foi o único que se destacou quanto ao caráter PR, em ambas as safras, e o genótipo BGMC 993 já se havia destacado, quanto a PR, em experimento conduzido por Vieira et al. (2013), em Planaltina-DF. A produtividade de raízes é um dos caracteres mais importantes para a seleção de genótipos de mandioca para indústria, em função de ser um dos fatores relacionados com os potenciais produtivos finais de farinha e de fécula de determinado genótipo, no local de realização dos experimentos (Fukuda et al., 2002; Ceballos et al., 2004).

Tabela 4: Comparação de médias dos caracteres peso da parte aérea, sem a cepa, em Kg ha' ${ }^{-1}$ (PPA), produtividade de raízes, em Kg $\mathrm{ha}^{-1}(\mathrm{PR})$, percentagem de amido nas raízes (AM) e rendimento de amido, em Kg ha-1 (RA), avaliados em 12 genótipos de mandioca para indústria, nas safras 2010/2012 (S1) e 2011/2013 (S2), em Unaí-MG

\begin{tabular}{lcccccccc}
\hline \multirow{2}{*}{ Acess os } & PPA & PPA & PR & PR & AM & AM & RA & RA \\
\cline { 2 - 9 } & S1 & S2 & S1 & S2 & S1 & S2 & S1 & S2 \\
\hline Clone 2/87 & $21215 \mathrm{fA}^{*}$ & $21521 \mathrm{cA}$ & $30771 \mathrm{cA}$ & $30993 \mathrm{bA}$ & $31,21 \mathrm{aA}$ & $30,73 \mathrm{bA}$ & $9616 \mathrm{cA}$ & $9521 \mathrm{bA}$ \\
Clone 2/88 & $19694 \mathrm{fA}$ & $20618 \mathrm{cA}$ & $31180 \mathrm{cA}$ & $31729 \mathrm{bA}$ & $31,63 \mathrm{aA}$ & $30,58 \mathrm{bA}$ & $9885 \mathrm{cA}$ & $9694 \mathrm{bA}$ \\
Clone 3/88 & $34028 \mathrm{cA}$ & $31062 \mathrm{aA}$ & $23198 \mathrm{~dB}$ & $27805 \mathrm{cA}$ & $29,77 \mathrm{bA}$ & $30,39 \mathrm{bA}$ & $6907 \mathrm{dA}$ & $8445 \mathrm{bA}$ \\
Clone 6/88 & $31486 \mathrm{dA}$ & $26361 \mathrm{bB}$ & $29629 \mathrm{cA}$ & $32153 \mathrm{bA}$ & $32,04 \mathrm{aA}$ & $31,21 \mathrm{bA}$ & $9497 \mathrm{cA}$ & $10046 \mathrm{bA}$ \\
Clone 10/87 & $16521 \mathrm{fA}$ & $13937 \mathrm{dA}$ & $26583 \mathrm{dA}$ & $30319 \mathrm{bA}$ & $29,88 \mathrm{bA}$ & $28,28 \mathrm{cA}$ & $7977 \mathrm{dA}$ & $8542 \mathrm{bA}$ \\
BGMC 436 & $20980 \mathrm{fA}$ & $20812 \mathrm{cA}$ & $27618 \mathrm{dA}$ & $29722 \mathrm{bA}$ & $31,33 \mathrm{aA}$ & $30,80 \mathrm{bA}$ & $8677 \mathrm{dA}$ & $9170 \mathrm{bA}$ \\
BGMC 1371 & $23062 \mathrm{fA}$ & $19104 \mathrm{cA}$ & $37639 \mathrm{bA}$ & $38479 \mathrm{aA}$ & $29,06 \mathrm{bA}$ & $28,39 \mathrm{cA}$ & $10936 \mathrm{bA}$ & $10915 \mathrm{aA}$ \\
BGMC 1304 & $40521 \mathrm{bA}$ & $27104 \mathrm{bB}$ & $27278 \mathrm{dA}$ & $25937 \mathrm{cA}$ & $30,73 \mathrm{aA}$ & $30,46 \mathrm{bA}$ & $8400 \mathrm{dA}$ & $7910 \mathrm{bA}$ \\
BGMC 991 & $51695 \mathrm{aA}$ & $34500 \mathrm{aB}$ & $39292 \mathrm{bA}$ & $36417 \mathrm{aA}$ & $27,54 \mathrm{bB}$ & $30,84 \mathrm{bA}$ & $10804 \mathrm{bA}$ & $11219 \mathrm{aA}$ \\
BGMC 993 & $27563 \mathrm{eA}$ & $21972 \mathrm{cB}$ & $31077 \mathrm{cA}$ & $35271 \mathrm{aA}$ & $31,84 \mathrm{aA}$ & $33,01 \mathrm{aA}$ & $9894 \mathrm{cB}$ & $11653 \mathrm{aA}$ \\
BGMC 994 & $24681 \mathrm{eB}$ & $29507 \mathrm{bA}$ & $38382 \mathrm{bA}$ & $39639 \mathrm{aA}$ & $31,49 \mathrm{aA}$ & $30,88 \mathrm{bA}$ & $12090 \mathrm{bA}$ & $12244 \mathrm{aA}$ \\
BGMC 996 & $42917 \mathrm{bA}$ & $33709 \mathrm{aB}$ & $56250 \mathrm{aA}$ & $37639 \mathrm{aB}$ & $32,29 \mathrm{aA}$ & $33,12 \mathrm{aA}$ & $18156 \mathrm{aA}$ & $12466 \mathrm{aB}$ \\
\hline Média & $29530 \mathrm{~A}$ & $25017 \mathrm{~B}$ & $33241 \mathrm{~A}$ & $33009 \mathrm{~A}$ & $30,73 \mathrm{~A}$ & $30,72 \mathrm{~A}$ & $10237 \mathrm{~A}$ & $10152 \mathrm{~A}$ \\
Amplitude** & 35174 & 20563 & 33052 & 13702 & 4,75 & 4,84 & 11249 & 4556 \\
\hline
\end{tabular}

* = médias seguidas pela mesma letra maiúscula na horizontal e minúscula na vertical pertencem ao mesmo grupo, a 5\% de probabilidade de erro, pelo teste de agrupamento de médias de Scott e Knott; ${ }^{* *}=$ diferença entre a maior e a menor média. 
Dentre os genótipos avaliados, apenas o BGMC 996 apresentou média de rendimento de amido (RA) significativamente superior à dos demais, na safra 2010/2012. Já na safra 2011/2013, os genótipos que apresentaram médias de RA significativamente superiores às dos demais foram BGMC 996, BGMC 994, BGMC 993, BGMC 991 e BGMC 1371 (Tabela 4). Apenas o genótipo BGMC 996 destacou-se em relação aos demais, em ambas as safras, quanto ao rendimento de amido. O rendimento de amido é, sem dúvida, o parâmetro mais importante quando da seleção de genótipos de mandioca para indústria para determinada região, uma vez que revela o potencial produtivo final dos genótipos e, por consequência, a rentabilidade da lavoura (Fukuda et al., 2002; Ceballos et al., 2004). Contudo, também devem ser considerados, no momento da recomendação de determinado genótipo para o cultivo, fatores como arquitetura, coloração da polpa das raízes, coloração da epiderme das raízes, resistência a pragas e doenças, entre outros (Fukuda et al., 2002; Ceballos et al., 2004).

Com base nos resultados obtidos, é possível afirmar que, a região de Unaí-MG apresenta potencial para o cultivo de mandioca, uma vez que no experimento foi obtida: i) média de produtividade de raízes superior a 33 $\mathrm{t} \mathrm{ha}^{-1}$, o que é muito superior à média de produtividade de raízes de mandioca na região do Cerrado, de $13 \mathrm{t} \mathrm{ha}^{-1}$ (IBGE, 2011), ii) média de teor de amido nas raízes superior a $30 \%$; iii) média de rendimento de amido superior a $10 \mathrm{t} \mathrm{ha}^{-1}$; e iv) média de produtividade de parte aérea superior a $25 \mathrm{t} \mathrm{ha}^{-1}$, o que pode ser considerado uma boa produtividade de forragem, uma vez que a parte aérea de mandioca apresenta em torno de $13 \%$ de proteína bruta (Souza et al., 2011).

O acesso BGMC 996, que corresponde ao clone 09 do programa de melhoramento da Embrapa Mandioca e Fruticultura Tropical, destacou-se em relação aos demais em função do desempenho agronômico apresentado, uma vez que apresentou, em ambas as safras, elevadas médias de produtividade de raízes, elevados teores de amido nas raízes e, como consequência, elevado rendimento de amido (Tabela 4). Ademais, o clone apresentou peso da parte aérea inferior apenas ao do genótipo BGMC 991, na safra 2010/2012, e PPA no grupo dos acessos mais produtivos na safra 2011/2013 (Tabela 4).

Os resultados obtidos neste trabalho são promissores no que diz respeito ao potencial da cultura na região. Entretanto, antes da recomendação de qualquer um dos genótipos avaliados para o plantio comercial, é necessária a validação de seus desempenhos em maior número de locais, por meio da metodologia da seleção participativa de variedades de mandioca (Fialho \& Vieira, 2011), com a qual seriam obtidos, além de dados agronômicos, informações relativas à preferência dos produtores.

\section{CONCLUSÕES}

Os genótipos avaliados apresentam características positivas para cultivo em Unaí (MG).

O genótipo BGMC 996 apresenta elevado desempenho agronômico, com destaque para as produtividades de parte aérea, produtividade de raízes e rendimento de amido em suas raízes de reserva.

\section{AGRADECIMENTOS}

Os autores agradecem à Empresa Brasileira de Pesquisa Agropecuária (Embrapa), à Fundação Banco do Brasil (FBB) e ao Conselho Nacional de Desenvolvimento Científico e Tecnológico (CNPq) pelo apoio financeiro.

\section{REFERÊNCIAS}

Borges MF, Fukuda WMG \& Rossetti AG (2002) Avaliação de variedades de mandioca para consumo humano. Pesquisa Agropecuária Brasileira, 37:1559-1565.

Ceballos H, Iglesias CA, Pérez JC \& Dixon AGO (2004) Cassava breeding: opportunities and challenges. Plant Molecular Biology, 56:503-516.

Cruz CD (2001) Programa genes: aplicativo computacional em genética e estatística. Viçosa, UFV. 648p.

Fialho JF \& Vieira EA (2011) Seleção participativa de variedades de mandioca na agricultura familiar. $1^{\mathrm{a}}$ ed. Planaltina, Embrapa Cerrados. $76 \mathrm{p}$.

Fialho JF, Sousa DMG \& Vieira EA (2013) Manejo do solo no cultivo de mandioca. In: Fialho JF \& Vieira EA (Eds.) Mandioca no Cerrado: orientações técnicas. $2^{\mathrm{a}}$ ed. Planaltina, Embrapa Cerrados. p.39-60.

Fialho JF \& Vieira EA (2013) Manejo e tratos culturais da mandioca. In: Fialho JF \& Vieira EA (Eds.) Mandioca no Cerrado: orientações técnicas. $2^{\text {a }}$ ed. Planaltina, Embrapa Cerrados. p.61-88.

Fialho JF, Vieira EA, Silva MS, Paula-Moraes SV, Fukuda WMG, Santos Filho MOS \& Silva KN (2009) Desempenho de variedades de mandioca de mesa no Distrito Federal. Revista Brasileira de Agrociência, 15:31-35.

Fukuda WMG, Silva SO \& Iglesias C (2002) Cassava breeding. Crop Breeding and Applied Biotechnology, 2:617-638.

Gomes FP (1991) Análise de grupos de experimentos. In: Gomes FP (Ed.) Curso de estatística experimental. 14ª ed. Piracicaba, Nobel. p.168-197.

Grosmann J \& Freitas AG (1950) Determinação do teor de matéria seca pelo método peso específico em raízes de mandioca. Revista Agronômica, 14:75-80.

IBGE - Instituto Brasileiro de Geografia e Estatística (2011) Sistema IBGE de recuperação automática - SIDRA. Disponível em: <http:// www.sidra.ibge.gov.br/> . Acessado em: 15 de outubro de 2013.

Oliveira MN, Xavier JHV, Silva FAM, Scopel E \& Zoby JLF (2009) Efeitos da introdução do sistema de plantio direto de milho por agricultores familiares do município de Unaí, MG (Cerrado Brasileiro). Pesquisa Agropecuária Tropical, 39:51-60.

Otsubo AA, Brito OM, Mercante FM, Otsubo VHN, Gonçalves MA \& Telles TS (2009) Desempenho de cultivares elites de mandioca industrial em áreas de cerrado do Mato Grosso do Sul. Semina: Ciências Agrárias, 30:1155-1162. 
Sagrilo E, Vidigal Filho PS, Otsubo AK, Silva AS \& Rohden VS (2010) Performance de cultivares de mandioca e incidência de mosca branca no Vale do Ivinhema, Mato Grosso do Sul. Revista Ceres, 57:8794

SAS Institute Inc. (2006) Statistical analysis system user's guide. Version 9.1. Cary, Statistical Analysis System Institute. 235p.

Souza AS, Rocha Júnior VR, Mota ADS, Palma MNN, Franco MO, Dutra ES, Santos CCR, Aguiar ACR \& Oliveira CR (2011) Valor nutricional de frações da parte aérea de quatro variedades de mandioca. Revista Brasileira de Saúde e Produção Animal, 12:441-455.

Vidigal Filho OS, Pequeno MG, Scapim CA, Vidigal MCG, Maia RR, Sagrilo E, Simon GA \& Lima RS (2000) Avaliação de cultivares de mandioca na região noroeste do Paraná. Bragantia, 59:69-75.

Vieira EA, Fialho JF, Faleiro FG, Bellon G, Fonseca KG, Carvalho LJCB, Silva MS, Moraes SVP, Filho MOSS \& Silva KN (2008) Divergência genética entre acessos açucarados e não açucarados de mandioca. Pesquisa Agropecuária Brasileira, 43:1707-1715.
Vieira EA, Fialho JF, Faleiro FG, Bellon G, Fonseca KG, Carvalho LJCB, Silva MS, Paula-Moraes SV, Oliveira CM \& Denke ML (2011) Characterization of sweet cassava acessions based on molecular, quantitative and qualitative data. Crop Breeding and Applied Biotechnology, 11:232-240.

Vieira EA, Fialho JF, Faleiro FG, Bellon G, Fonseca KG, Silva MS, PaulaMoraes SV \& Carvalho LJCB (2013) Caracterização fenotípica e molecular de acessos de mandioca de indústria com potencial de adaptação às condições do cerrado do Brasil Central. Semina: Ciências Agrárias, 34:567-582.

Vieira EA, Fialho JF, Silva MS, Fukuda WMG \& Santos Filho MOS (2009) Comportamento de genótipos de mandioca de mesa no Distrito Federal. Revista Ciência Agronômica, 40:113-122.

Vilpoux OF (2008) Competitividade da mandioca no Brasil, como matéria prima para amido. Informações Econômicas, 38:27-38.

Xavier JHV, Caldeira-Pires A, Zoby JLF \& Gastal ML (2005) Análise de ciclo de vida (ACV) de sistemas de produção da agricultura familiar em Unaí, MG: Resultados econômicos e impactos ambientais. Cadernos de Ciência \& Tecnologia, 22:547-586. 